\title{
Interpolating an Unorganized 2D Point Cloud with a Single Closed Shape
}

\author{
Stefan Ohrhallinger ${ }^{1}$, Sudhir P. Mudur ${ }^{2}$
}

\begin{abstract}
Given an unorganized two-dimensional point cloud, we address the problem of efficiently constructing a single aesthetically pleasing closed interpolating shape, without requiring dense or uniform spacing. Using Gestalt's laws of proximity, closure and good continuity as guidance for visual aesthetics, we require that our constructed shape be minimal perimeter, non-self intersecting and manifold. We find that this yields visually pleasing results. Our algorithm is distinct from earlier shape reconstruction approaches, in that it exploits the overlap between the desired shape and a related minimal graph, the Euclidean Minimum Spanning Tree (EMST). Our algorithm segments the EMST to retain as much of it as required and then locally partitions and solves the problem efficiently. Comparison with some of the best currently known solutions shows that our algorithm yields better results.
\end{abstract}

Keywords:

computational geometry, reconstruction, construction, shape, curve, boundary, point cloud, point set, EMST

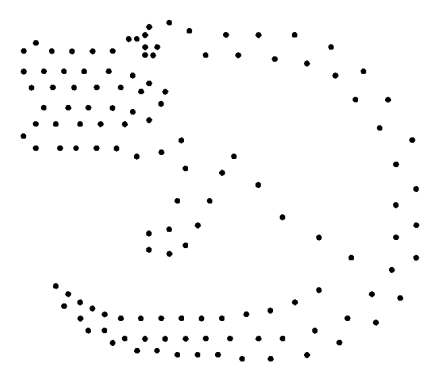

a)

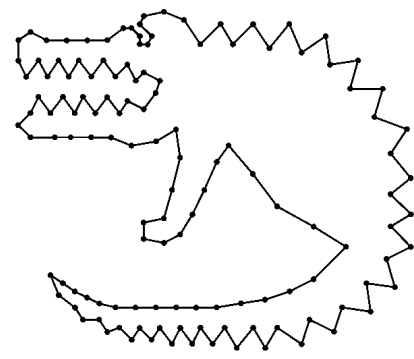

b)
Figure 1: a) A sparsely sampled unorganized point set. b) Its closed manifold interpolating boundary with minimum perimeter.

\section{Introduction}

The goal is to identify a single aesthetically pleasing shape connecting points in a $2 \mathrm{D}$ unorganized point set, without requiring dense or uniform spacing. To fulfil the aesthetic requirement, we use for guidance Gestalt's laws of proximity (human tendency to connect close dots), closure and good continuity (smoothness) [27] to obtain measurable and objective criteria. Accordingly we require that the shape be a closed, nonselfintersecting manifold (law of closure) which interpolates all the points with minimum length (law of proximity), henceforth denoted by $S_{\text {min }}$. For the law of good continuity we will present a further constraint below. If we exclude extreme point distributions from our problem domain, the algorithm presented in this paper provides an efficient solution (see Figure 1).

The task of 2D shape reconstruction from boundary sampled points plays an especially important role in a number of engineering fields: reverse engineering of geometric models, outline reconstruction from feature points in medical image analysis, etc. The closed boundary is essential for calculating various shape moments, a characteristic property with many applications.

\section{Related Work}

Polygons interpolating a point set are also a topic in computational geometry, but the focus there is mostly on investigating lower and upper bounds on the total number of interpolating polygons based on the size of the given point set, say, as in [10].

If we consider all the methods in the literature for 2D shape reconstruction, they can be classified according to two major approaches which are discussed further below.

\subsection{Reconstruction with a Local Sampling Condition}

Early methods worked only on smooth and uniformly sampled point sets, such as $\alpha$-shapes [8, 16], Figueiredo and Gomes [17], $\beta$-skeleton [24], $\gamma$-neighborhood graph [30] and r-regular shapes [6]. For example, $\alpha$-shapes requires userspecification of a global constant which depends on sampling. It does not work for non-uniformly sampled point sets. It also cannot guarantee a manifold the way our algorithm does.

Amenta et al. [3] with their Crust algorithm introduced the concept of local feature size which allows reconstruction from non-uniformly sampled point sets. The stated sampling requirements of the Crust method and its successors [11, 12] are however quite restrictive in theory and difficult to ensure in practice. Not only is it difficult to check if a given point cloud satisfies the sampling requirement, but it is even more difficult to construct a sampling satisfying the requirement. It should be noted though that the presented algorithms often show reconstruction of less restricted point sets but with no guarantees. DIS$C U R$ [31] uses the two properties of proximity and smoothness but still requires rather dense sampling in sharp corners. Some improvements on these aspects have been made in VICUR [28], 
but it relies very much on user-tuned parameters and regresses for other point sets. The Gathan algorithm from Dey et al. [13] also handles sharp corners, but again without guarantees. Gath$a n G$ [14] is an extension which, like our work, is targeted at closed shapes and gives guarantees exclusively for certain conditions. It still does not work for many cases we tested. In spite of this, it provides in our opinion the best solution to date for this $2 \mathrm{D}$ shape reconstruction problem.

All of the above-mentioned algorithms reconstruct a boundary using edges in the Delaunay Graph $(D G)$ and results have shown that this is a very reasonable choice. The $D G$ has the property of maximizing its angles and minimizing its edge lengths, which conform to the Gestalt laws of good continuity and proximity. A minimum boundary which is not constrained to $D G$ may trade in longer edges and sharper angles instead.

A fundamental advantage of our method versus using a local criterion is that we can achieve far superior results for reconstruction of the single closed manifold shape which we require, for the particular subclass of point sets which represent such a shape. Instead the output of the previous methods only partially reconstructs such a shape as one or more open curves or as a number of ambiguous shapes (Figure 13 shows a number of such cases).

\subsection{Construction as Global Minimization of a Criterion}

Finding the minimum perimeter closed boundary actually requires a global search of the solution space.

A first attempt on global construction presented in [20] finds spanning Voronoi trees and selects the one with minimal length by integer programming, with $\mathrm{O}\left(n^{2} \log \mathrm{n}\right)$ complexity. It does not work well for sharp angles and non-uniform sampling; obviously it prunes good solutions too early.

Giesen shows in [19] that the exact solution to the travelling salesman problem (TSP) can reconstruct the shape for sufficiently dense sampling. Althaus et al. extend this work in [1] to non-uniform sampling with some conditions, and in [2] they compare it with both the Crust-type family of algorithms and TSP-approximations. They note that the latter two methods fail for certain curves with sparser sampling which the exact TSP method handles well. They also mention that the exponential complexity of the TSP decreases with denser sampling. With the exception of [19], these methods do not require userspecified parameters. Unfortunately, finding the exact solution using the TSP approach takes unreasonable time $\mathrm{O}\left(2^{n}\right)$ even for small $P$. The concorde exact TSP solver [4] scales subexponentially and can take hundreds of CPU-years for mediumsized point sets. A detailed discussion on its complexity is available in [23].

TSP approximations show more reasonable complexity but are not linearithmic, i.e. $O\left(n^{2.2}\right)$ [22] or $O\left(n(\log n)^{O(c)}\right)$ for a $(1+1 / c)$ approximation of the optimal tour of an Euclidean TSP [5], and $O\left(n^{O(1 / \epsilon)}\right)$ for $(1+\epsilon)$ times the solution for a planar graph TSP [21]. More importantly, they fail to guarantee the minimum solution and even a single wrongly connected edge may have a significant impact on aesthetic quality of the reconstruction. Hence approximation schemes for TSP cannot guarantee the desired interpolating and manifold shape.
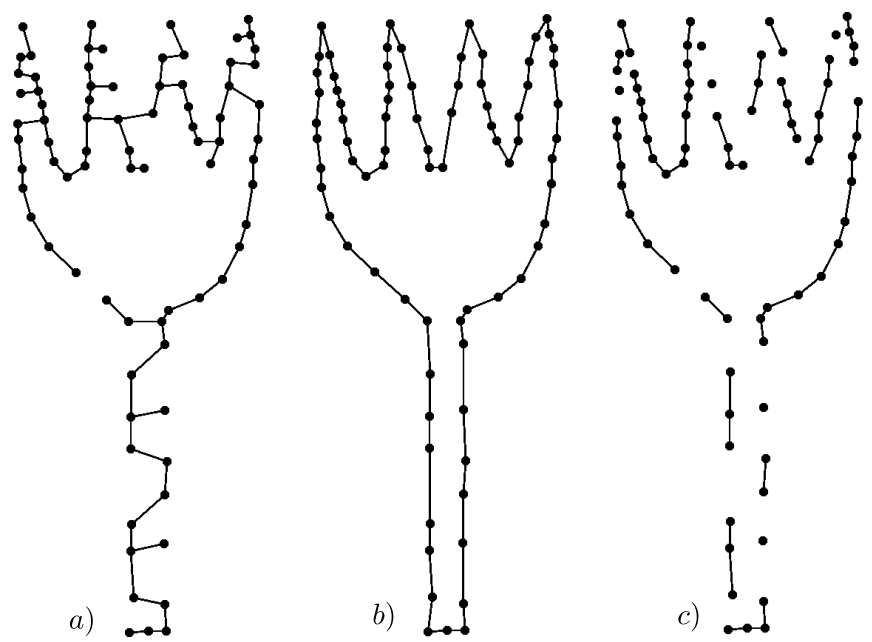

Figure 2: $E M S T$ and $S_{\text {min }}$ have considerable overlap even for sparsely sampled point sets like the tulip: a) $E M S T$ has 78 edges. b) $S_{\min }$ has 79 edges. c) 57 of their edges are shared.

While we too impose the minimum perimeter requirement, by restricting the sub-domain of that problem to edges in $D G$, we exploit the relationship between the Euclidean minimum Spanning Tree (EMST) and $S_{\min }$ (constrained to $D G$ ). Our algorithm segments the $E M S T$ and classifies the segments so as to retain as many of them as possible in the reconstruction of $S_{\text {min }}$. This way we partition the problem and provide an efficient solution. $S_{\text {min }}$ differs from $E M S T$ only by restricting its vertices to be manifold, which in turn increases its length (see Figure 2).

This relationship between the minimum spanning tree and the shape has been mentioned in Figueiredo and Gomes [17]. However, they only prove reconstruction for very densely sampled point sets: an EMST without branches. They do suggest some parameter-based heuristics for more sparsely sampled point sets, but do not really exploit this relationship in the unique way we do in our algorithm. We show that our algorithm can quickly find the desired solution and scales well to handle very large point sets. And if we exclude extremely sparse and highly non-uniformly sampled point sets, our algorithm's complexity is just $\mathrm{O}(\mathrm{n} \log \mathrm{n})$. While we do note that a constrained TSP solution restricted to the planar graph $D G$ would yield the same result, i.e., $S_{\text {min }}$, we are not aware of any TSP solution with this performance.

\subsection{Intuitive Overview of our Method}

Our method starts from a Delaunay graph $(D G)$ and the Euclidean minimum spanning tree (EMST) of the point set (see Figures $3 \mathrm{a}$ and $3 \mathrm{~b}$ ). EMS T is a subset of $D G$ (Attene and Spagnuolo [7]) and can be constructed in $O(n \log n)$ (Kruskal [25]).

It can have a number of non-manifold vertices with degree not equal to 2 (leaf or fork vertices). To such vertices we apply edge exchange operations like those used in the degreeconstrained spanning tree problem [29]. Determining this set of operations so that EMST is transformed into $S_{\text {min }}$ is NPhard. Our main contribution in this paper is an innovative way 


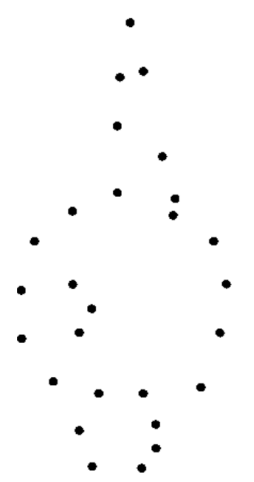

a)

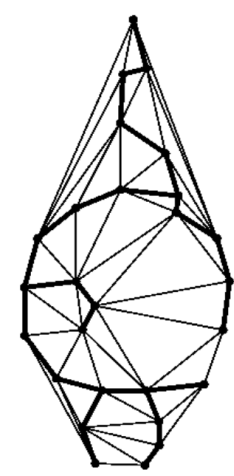

b)

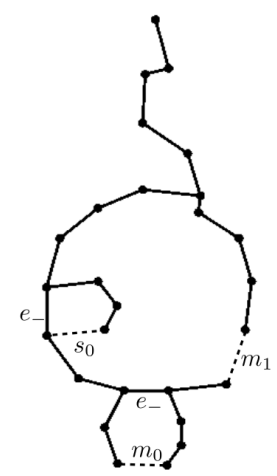

c)

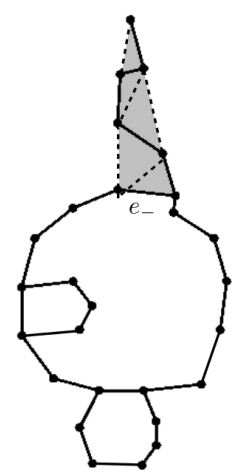

d)

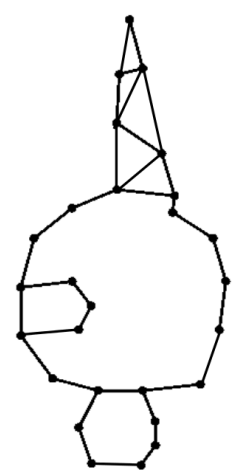

e)

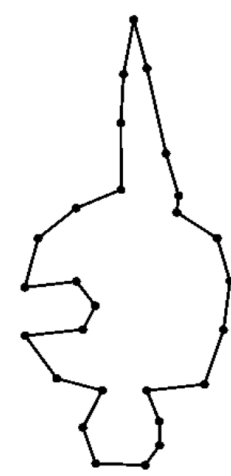

f)

Figure 3: a) Example point set $P$. b) $D G$ with edges in $E M S T$ emphasized. c) Edges incident to leaf vertices ( $m_{0}, m_{1}$ and $\left.s_{0}\right)$ shown with dotted lines. d) $E M S T$ with edges added and envelope of inflatable branch shaded with its edges not in EMST shown with dotted lines. e) Envelope edges added. f) Removal of cut edges yields $S_{\min }$.

of efficiently performing these edge exchange operations.

The other steps in our algorithm are the following:

- Segment the EMST at fork vertices and retain as many segments as possible, since such segments are already of minimum length (Figure 3c).

- Adding an edge in $D G$ to the EMST graph creates a loop. If two loops share a single edge called as cut edge, then deleting just the cut edge results in a single loop interpolating points in both loops. On the other hand if two loops share a single vertex or an edge-chain, then removal of these shared vertices will result in either a split graph or vertices which are not interpolated. Hence, we only add edges which lead to loops sharing a cut edge. The next step is therefore to select and add the $D G \backslash E M S T$ edges, incident to leaf vertices, needed to make $S_{\min }$.

- The resulting graph will have the following configuration: (i) a single loop or multiple loops connected by pairs of loops sharing cut edges, and (ii) segments (like strands) of EMS T connected to a loop at one end and open or connected to another loop at the other end (Figure 3d).

- In the next step the strand-like segments are converted (inflated) into loops and then all cut edges are removed to yield a manifold interpolating shape (Figure 3d-f).

In the following sections we present these steps in detail and further identify the point configurations for which our algorithm is guaranteed to work.

\section{Definitions}

Closed shape $S$ is a single manifold polygon interpolating all $v_{i} \in$ point set $P$ and consisting of edges $e_{i} \in$ Delaunay graph $D G$ of $P .\left\{S_{i}\right\}$ denotes the set of all such closed shapes in $P$ and $S_{\text {min }}$ denotes the one with minimum perimeter.

Hamiltonian graph is a graph $G=(V, E)$ with at least one closed shape $S$ (Dillencourt [15]). Genoud [18] shows that $D G$ for any $P$ is rarely non-Hamiltonian.

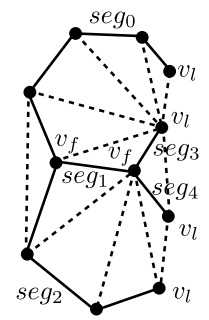

a)

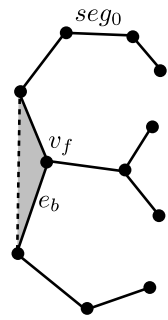

b)

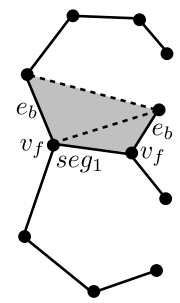

c)

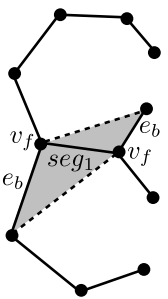

d)
Figure 4: EMST with other edges in $D G$ shown dotted: a) Segments labeled as $\operatorname{seg}_{i}$, leaf vertices as $v_{l}$, fork vertices as $v_{f}$. $s e g_{1}$ is a trunk segment, all other segments are branches. b) One of two non-manifold envelope boundaries of $\operatorname{seg}_{0}$ (shaded grey) with base edge $e_{b} . s g_{0}$ is therefore a retained segment. c) First manifold envelope boundary of $\operatorname{seg}_{1}$ with two fork vertices and a base edge each. $\operatorname{seg}_{1}$ is therefore a non-retained segment. d) Second manifold envelope boundary of $\operatorname{seg}_{1}$; the other two envelope boundaries are non-manifold.

\section{Loop is a cyclic sequence of edges.}

Segment $s$ is a sequence of manifoldly connected edges terminated by non-manifold vertices (with degree $\neq 2$ ), either leaf vertices $v_{l}$ (degree 1 ) or fork vertices $v_{f}$ (degree $>2$ ). A segment with at least one leaf vertex is called a branch $b$. All other segments are trunk segments (see Figure 4 for examples).

$C u t$ is the set of vertices of a connected graph $G$ whose removal renders $G$ disconnected. A cut edge has two cut vertices and a cut edge-chain has more than two.

Uniformity of sampling $u=d_{i} / d_{j}$ where $d_{i}, d_{j}$ are the Euclidean distances between a point $p \in P$ and its neighbors in $S_{\text {min }}$, sorted such that $d_{i}>d_{j} . u_{\max }$ is then the largest $u$ for any $p \in P$. The larger $u_{\text {max }}$ is, the less uniform is $P$.

Sharp angled features: In [3] the notion of Local Feature Size was introduced, primarily for smooth shapes (no sharp corners), which depends on local curvature and proximity. They state: For an $r$-sampled curve in the plane, $r<1$, the angle spanned by three adjacent samples is at least $\pi-4 \arcsin (r / 2)$. This condition does not evaluate for $r>=1$, therefore it cannot support angles $<=60^{\circ}$. Since our method can handle much sharper angles, we use instead $\alpha_{\text {min }}$, the minimum angle between any three adjacent points in the desired closed shape as a 
measure of sharp features.

\section{Defining Operations}

\subsection{Segment Classification}

We classify EMST segments into those which are part of $S_{\min }$ and those which are not. This classification is based on the observation that for retained segments, any other edge sequence connecting the segment's interior vertices (all except the end vertices) will either result in an increase in length or will not be manifold.

Base edge $e_{b}$ for a segment $s$ is defined as follows. Let the edge $e_{i} \in s$ be incident to a fork vertex $v_{f} \in s$. Then two base edges $e_{b}$ are the immediately adjacent edges in $c w$ and $c c w$ sense, incident to $v_{f}$. It may be noted that a trunk segment has four base edges, a branch with one leaf vertex has two and a branch with both ends as leaf vertices has none. The vertex of $e_{b}$ opposite to $v_{f}$ is called a base vertex $v_{b}$.

Envelope env $(s)$ for a segment $s$ is the set of Delaunay triangles for which the vertices consist of the vertex set $\epsilon s$ and one base vertex per fork vertex. The envelope boundary may or may not be manifold. A branch has two envelopes and a trunk has four.

Retained segment is a segment for which none of its envelope boundaries is manifold. For such segments, there is no alternative way of manifoldly interpolating the segment vertices without increasing their length. All other segments are non-retained (see Figure 4 for both cases).

\subsection{Inflate and Select Minimum Loop Operation}

A non-retained segment for which a manifold envelope boundary exists is a strand which may require to be modified to become part of the desired closed shape. A manifold envelope boundary can always be modified to form a loop which interpolates all the vertices in that segment. There may be a number of different choices for forming these interpolating loops. We select the minimum length one. We call this as the inflate operation associated with the segment. Since all loops of a segment share its base edges with the remainder of $S_{i}$, this operation corresponds to solving the reconstruction problem locally, i.e., segment-wise.

\subsection{Edge Displacement Operations}

$S_{\text {min }}$ for a point set with $n$ points has $n$ edges, while its $E M S T$ has one edge less since it is a tree.

Let $E_{+}=S_{\text {min }} \backslash E M S T$ denote the set of edges that we need to add to $E M S T$ when transforming it into $S_{\text {min }}$. Note that the same number of edges minus 1 must be removed from $E M S T$ to maintain the edge count.

A subset of potential edges in $E_{+}$is easily identifiable. In this subset edges are incident to leaf vertices. Each of them forms a loop when added to EMST. We classify the operations of adding edges into two types of edge displacement operations (see Figure 5 for examples):

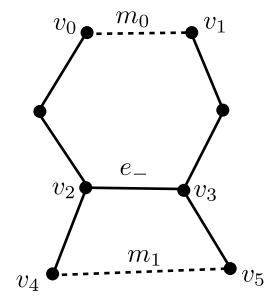

a)

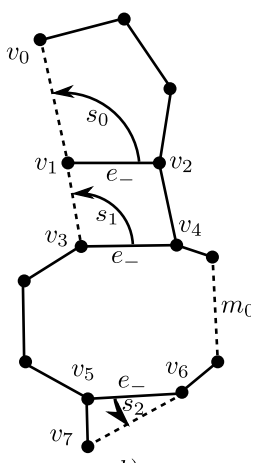

b)
Figure 5: a) Example of EMST with moves: $m_{0}\left(v_{0}, v_{1}\right)$ and $m_{1}\left(v_{4}, v_{5}\right)$ both associated with the trunk segment $e_{-}\left(v_{2}, v_{3}\right)$. b). Another example of EMST with move and snaps: $s_{0}\left(v_{1}, v_{0}\right)$ with $e_{-}\left(v_{1}, v_{2}\right)$ has two leaf vertices, $s_{1}\left(v_{3}, v_{1}\right)$ with $e_{-}\left(v_{3}, v_{4}\right)$ and $s_{2}\left(v_{6}, v_{7}\right)$ with $e_{-}\left(v_{6}, v_{5}\right)$. For $s_{0}: v_{-}=v_{0}, v_{=}=v_{1}, v_{+}=v_{2}$.

- move edge $e_{+}$: between two leaf vertices. For satisfying the manifold condition, there has to exist a corresponding edge $e_{-} \in E M S T$ incident to a fork vertex in the move's loop. We say that $e_{-}$moves to $e_{+}$since the two edges do not share any vertices. Let us recall that EMST has one edge less than $S_{\min }$. Therefore there will exist one move without $e_{-}$.

- snap edge $e_{+}$: incident to a leaf vertex $v_{-}$. Its other vertex $v_{=}$can be a leaf or a manifold vertex. $e_{-}\left(v_{=}, v_{+}\right)$is the edge incident to a fork vertex $v_{+}$in the snap's loop. We say that $e_{-}$snaps from $v_{+}$about $v_{=}$to $v_{-}$, to become $e_{+}$.

We want to underline that only a subset of the move and snap operations identified this way will actually need to be applied. Therefore we will use the term candidate for them in the context where they are just potential operations, as opposed to when they have been definitely applied as operations.

The $e_{-}$edge of a snap candidate can be locally identified. The $e_{-}$edge of a move could be anywhere in its loop, and in principle entails a global search. However, as mentioned earlier, we ingeniously avoid this global search, by clustering together all the add edge operations $\left(e_{+}\right)$and then removing all the corresponding $e_{-}$edges at once, as they are all identifiable as cut edges.

\subsection{Associations of Candidates to Segments}

In order to decide which of the candidate edges should be added to make $S_{\text {min }}$, we create a segment - candidate association table and then evaluate the candidate's applicability based on three conditions, interpolation, manifold and minimum length. This table enables the evaluation of all potential solutions. One column indicates the type (retained/non-retained) and another distinct column lists all the candidates associated with each segment in EMST.

Every segment entirely contained in the loop created by the addition of a candidate edge is said to be associated with it and vice versa. The only exception is the segment containing edge $e_{-}$in the loop formed by a snap edge $e_{+}$, since this $e_{-}$is 


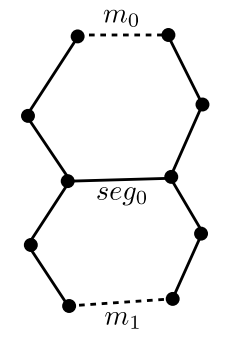

a)

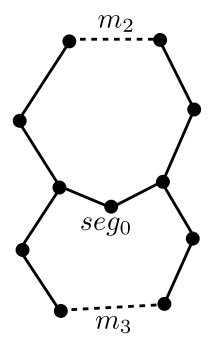

b)
Figure 6: a) Single-edge trunk segment $\operatorname{seg}_{0}$ can be contained in the loops of two candidates $m_{0}, m_{1}$ since it forms a cut edge. b) Candidates $m_{2}, m_{3}$ can not both be permitted to be applied for multiple-edge trunk segment $s e g_{0}$ : since its removal will disconnect $v_{0}$.

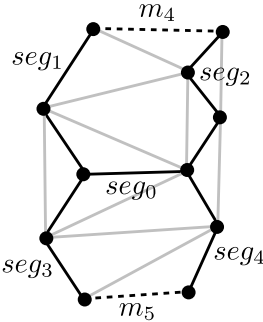

a)

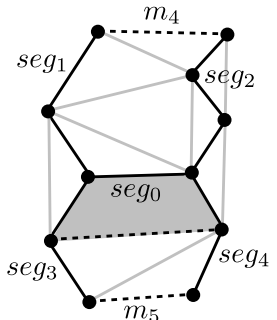

b)

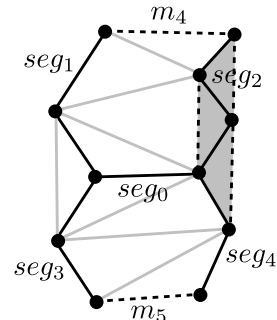

c)
Figure 7: a) $E M S T$ of a point set with $D G$ edges shaded grey and segments and candidates marked. b) manifold envelope of $s g_{0}$ shaded. c) manifold envelope of $\operatorname{seg}_{2}$ shaded.

removed in a subsequent step. Let us note that this segmentcandidate association is many-to-many, see for example Figure $6 \mathrm{~b}$, in which $\operatorname{seg}_{0}$ is contained in the loops of two move candidates $m_{0}$ and $m_{1}$. Of course, we can only apply one of them while respecting the condition of a manifold boundary.

\subsection{The Solution Space Tree}

A set of candidates associated with a segment is said to be multi-choice if not all of them can be applied simultaneously. Table 1 shows this for the example point set shown in Figure 7. In this example, trunk $\# 0$ and branch $\# 2$ are the multi-choice segments. Using such a table, we can explore all potential solutions by viewing the solution space as a tree. Each candidate associated with a multi-choice segment represents a branching point. Each terminal node contains a potential solution with a permissible subset of candidates per segment. The size of the solution space tree is the product of its multi-choice candidate set sizes. So this example contains $3 * 2=6$ potential solutions.

\subsection{Pruning of the Solution Space Tree}

It is easy to see that the solution space tree can grow quickly. For efficient searching, our algorithm prunes parts of this tree as early as possible by eliminating any candidates leading to a non-manifold solution. In fact it dynamically constructs the solution tree, doing the branching required for exploring new solutions only where it is unavoidable. If a candidate is the only one associated with a segment, then it is applied. This in turn could lead to reducing the number of candidates in other

\begin{tabular}{|l|l|l|}
\hline Segment & Segment type & Candidate Ops \\
\hline trunk \#0 & non-retained & $m_{4} m_{5}$ inflate \\
branch \#1 & retained & $m_{4}$ \\
branch \#2 & non-retained & $m_{4}$ inflate \\
branch \#3 & retained & $m_{5}$ \\
branch \#4 & retained & $m_{5}$ \\
\hline
\end{tabular}

Table 1: Segment-candidate table for point set of Figure 7.

multi-choice segments, which may result in more such single candidates. Thus evaluation of multiple solutions is only necessary when we are left with nothing but multi-choice segments in the table.

We shall see in the examples later that even in problems with very large solution spaces, this procedure of eliminating candidates is very effective and typically results in construction of only a small part of the solution tree. This is what makes this algorithm efficient. More details of the algorithm follow, giving all the states when candidates can be applied, eliminated and when multiple solutions have to be evaluated.

\section{Algorithm}

1. For a given point set $P$, create $D G$ and $E M S T$, segment EMST and classify segments as retained or non-retained.

2. Identify candidate operations and create segmentcandidate association table.

3. Initialize $E_{\text {curr }}=E M S T$.

4. Apply an applicable move or snap candidate from the segment-candidate association table. A candidate is not applicable if it results in a non-manifold condition, namely, a cut vertex, a cut edge-chain or its $e_{-}$causes a loop to become open.

5. Prune the segment-candidate association table by eliminating all associations of invalidated candidates as follows. As a result of the previous add edge operation, some segments will already be part of a loop; their associated candidates are no longer needed and are removed from the table. Some of the leaf vertices will become manifold; candidates incident on such vertices are also removed from the table. Lastly, any candidate which if applied will lead to a non-manifold boundary is also removed.

6. Repeat steps 5 and 6 (Apply and Prune) until there are no more move or snap operations left.

7. Carry out inflate operations remaining in the table.

8. Remove cut edges in $E_{\text {curr }}$

The above are the major steps in our algorithm. In the implementation there is detailed case analysis based on the type of operation move or snap for detecting the non-manifold condition.

Once the association table contains only multi-choice segments, we select the segment with least number of candidates, and explore all the solutions. The order in which candidates are applied (and thus the order in which the solution space is traversed) does not matter. This is because our algorithm evaluates all potential solutions. 


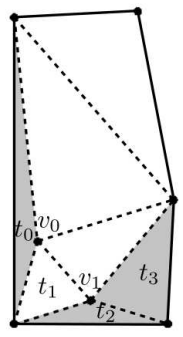

a)

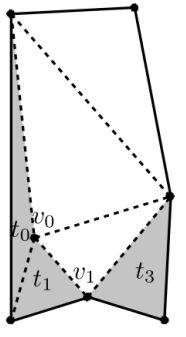

b)

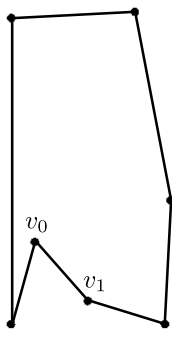

c)

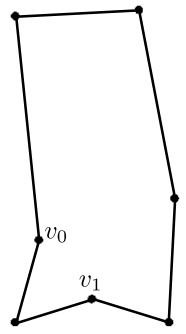

d)
Figure 8: A manifold envelope boundary env $v_{0}$ (shown using solid lines) with two interior vertices: a) shows triangles $t_{0}, t_{2}$ and $t_{3}$ for removal from env $v_{0}$. b) $e n v_{1}=e n v_{0} \backslash t_{2}$ : new removal triangles are $t_{0}, t_{1}$ and $t_{3}$. c) $e n v_{2}=e n v_{0} \backslash\left\{t_{2}, t_{1}\right\}$ : one potential local loop since no interior vertices remain. d) Final minimum length local loop for the example: $e n v_{3}=e n v_{0} \backslash\left\{t_{0}, t_{2}\right\}$

\subsection{Inflate Operation}

As already mentioned, we will need to apply the inflate operation to all the connected sets of remaining segments (inflatable sub-trees) which do not form part of any loop. This is described next. Triangles with a single edge on the envelope's boundary and one vertex in the interior are placed in a list and removed until no interior vertices are left. This will yield a loop interpolating all the vertices in the segment. The loop causing minimum increase in perimeter of $S_{\min }$ is chosen (see Figure 8).

\subsection{Remove Cut Edges}

After all the inflate operations are carried out, there are no more operations in the segment-candidate association table to apply. $E_{\text {curr }}$ will have a number of loops connected to each other through cut edges, which have to be detected and deleted to yield an interpolating manifold boundary. A generic algorithm to detect cut edges in a graph is of higher than linear (worst case) complexity. But we can do this with linear time worst case complexity by exploiting the knowledge we have about the applied candidates as follows:

We know that the cut edges of snaps are their $e_{-}$and the ones of the inflates are inside their chosen modified envelope. The remaining cut edges are associated with moves. These are not known but do not overlap among each other.

Therefore we just have to first remove all cut edges resulting from application of snaps and inflates and then remove all edges between vertices of degree $>2$.

\subsection{Examples with multi-choice segments}

The segment-candidate shown in Table 2 for our first example (a figure-eight), has all multi-choice segments, except branch \#2 (Figure 9. The Apply-Prune steps leading to the solution are also shown.

Let us briefly follow the progress of our algorithm for this example. $m_{2}$, the only operation associated with branch \#2 is first applied. Consequently $s_{1}, m_{3}$ and $s_{6}$ get eliminated. $m_{5}$, the only operation associated with branch \#4 is applied next. All remaining operations get eliminated, resulting in the desired shape.

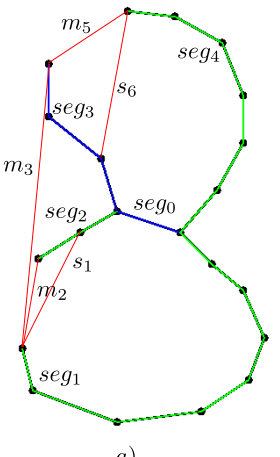

a)

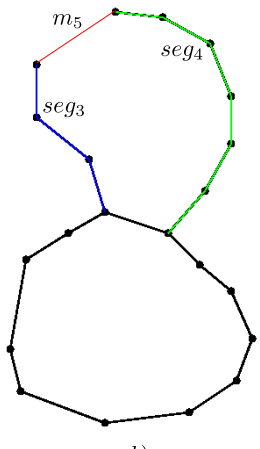

b)

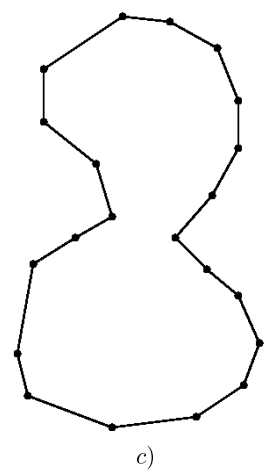

Figure 9: a) EMST with segments (blue: non-retained, green: retained) and candidates (red) marked. b) Applying $m_{2}$ creates a loop in the lower half and leaves only one candidate $m_{5}$. c) Applying $m_{5}$ and removing the cut edge yields a single $S_{i}$, which is the desired solution.

\begin{tabular}{|l|l|l|l|l|}
\hline Segment & Type & Candidate Ops & $m_{2}$ applied & $m_{5}$ applied \\
\hline trunk \#0 & non-retained & $s_{1} s_{6} m_{2} m_{3} m_{5}$ inflate & $\mathbf{m}_{\mathbf{2}} m_{5}$ inflate & $\mathbf{m}_{\mathbf{2}} \mathbf{m}_{\mathbf{5}}$ \\
branch \#1 & retained & $s_{1}, m_{2}, m_{3}$ & $\mathbf{m}_{\mathbf{2}}$ & $\mathbf{m}_{\mathbf{2}}$ \\
branch \#2 & retained & $m_{2} \leftarrow$ & $\mathbf{m}_{\mathbf{2}}$ & $\mathbf{m}_{\mathbf{2}}$ \\
branch \#3 & non-retained & $m_{3} m_{5}$ inflate & $m_{5}$ inflate & $\mathbf{m}_{\mathbf{5}}$ \\
branch \#4 & retained & $s_{6} m_{5}$ & $m_{5} \leftarrow$ & $\mathbf{m}_{\mathbf{5}}$ \\
\hline
\end{tabular}

Table 2: Progression in segment-candidate table (right-most column is solution). Candidate to apply next is marked by arrow.
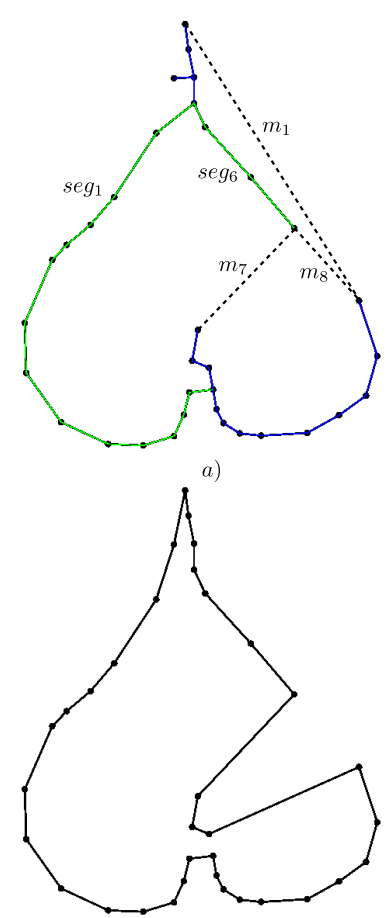

c)

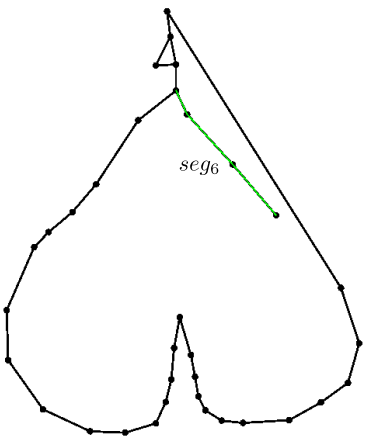

b)

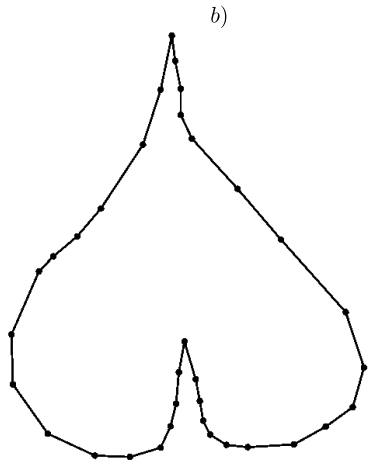

d)
Figure 10: a) EMST for point set from [31] with segments marked: green $=r e$ tained , blue $=$ non-retained . The multiple choice of candidates at $\operatorname{seg}_{1}$ creates three potential solutions $P S_{i}$ : b) $P S_{0}$ applies $m_{1}$ : in the end $\operatorname{seg}_{6}$ remains without candidates therefore $P S_{0}$ becomes invalid. c) $P S_{1}$ applies $m_{7}$ and produces $S_{0}$. d) $P S_{2}$ applies $m_{8}$ and produces $S_{1}$ which is $S_{\text {min }}$. 


\begin{tabular}{|l|l|l|}
\hline Segment & Type & Candidate Ops \\
\hline trunk \#0 & non-retained & $m_{0} m_{1} s_{4}$ loop \\
trunk \#1 & retained & $m_{1} m_{7} m_{8}$ \\
branch \#2 & non-retained & $m_{0} m_{1} s_{2}$ \\
branch \#3 & non-retained & $s_{2} s_{3} s_{4}$ \\
branch \#4 & non-retained & $m_{6} m_{7}$ loop \\
branch \#5 & non-retained & $m_{1} m_{6} m_{8} s_{9}$ \\
branch \#6 & retained & $m_{0} m_{7} m_{8}$ \\
\hline
\end{tabular}

Table 3: Initial segment-candidate table.

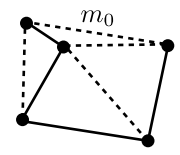

a)

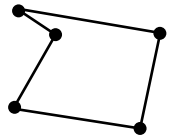

b)

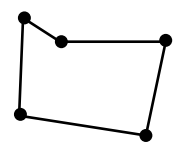

c)

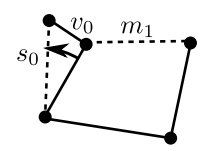

d)
Figure 11: a) EMST of non-conforming point set, with single operation $m_{0}$. b) Result is $S_{0}$ which is not minimal. c) $S_{\min }$. d) Therefore $m_{1}$ and $s_{0}$ are the correct operations to obtain $S_{\min }$ as their vertex degree changes cancel out at $v_{0}$ but they are not detectable.

\subsection{Examples with several potential solutions}

For the point set shown in Figure 10 all are multi-choice segments (see Table 3). The complete solution tree would have $4 * 3 * 3 * 3 * 3 * 4 * 3=3888$ terminal nodes (potential solutions). However, using our algorithm, very large parts of this solution tree get pruned and only a small number of potential solutions have to be actually evaluated to get $S_{\min }$.

The algorithm chooses the first segment with the minimum number of (all applicable) associated candidates: $\operatorname{seg}_{1}$. It branches into three potential solutions as shown in Figure 10. All other parts of the solution tree get pruned, so no further solutions need to be explored. Thus the total size of the search remains at 3 .

\subsection{Conforming Point Set Configurations}

Like most other algorithms, dense, uniformly sampled point sets are rather easily handled by our algorithm; this is also evident from the proof given in [17]. For such dense point configurations, the EMST already shares most edges with the desired boundary and the solution space tree remains small. As example we show a fairly large point set derived from a silhouette in Figure 12e. Noisy data is also interpolated as long as shape features are not significantly affected. If the noise is such that the point spacing gets unreasonably non-uniform, then the algorithm will terminate with an incomplete or incorrect result. For such noisy data an approximation algorithm like the one presented in [26] should be preferred. If one looks closely at the statistics in Table 4, those point sets which are densely sampled (Figure 12e) are relatively less complex to reconstruct than sparsely sampled sets.

Below we shall define the class of point configurations for which our algorithm can guarantee a minimum length boundary shape and give the proof for this. In a subsequent section we derive the computational complexity of our algorithm.

Our algorithm can guarantee the result for point set configurations in which edges in all required moves and snaps operations are connected to leaf vertices in EMST. Hence our algorithm requires that the input point set satisfy the following condition:

move and snap operations must not overlap such that a $e_{+}$and a $e_{-}$are incident to the same vertex. While such an overlap does not violate the manifold condition, the operations themselves are individually non-detectable (see an example in Figure 11).

We denote point sets satisfying the above condition as the conforming class.

Theorem 1. Our algorithm always terminates for point sets in the conforming class and produces their $S_{\text {min }}$.

Proof 1. All edges in retained segments of EMST, excepting the edges at each end, are guaranteed to be in $S_{\text {min }}$. Since the vertices of these edges are manifold, they do not permit any add edge operation without creating a non-manifold result. This proves that these edges do belong to $S_{\text {min }}$. For inflatable segments all interpolating loops through their vertices are evaluated and the minimal one selected. Finally, by the above condition, all remaining edge exchange combinations are detected and evaluated, and the combination yielding the manifold closed shape with minimal length is chosen.

In our experience the conforming class includes most sampled point sets encountered in practice, as they are usually dense and uniform. It also includes point configurations that are considerably more non-uniform and sparse. Further, in practice, for many point sets outside the conforming class, the algorithm will terminate and produce an interpolating shape, which is also aesthetic. However the above guarantee does not apply. We suggest later an extension to the algorithm to include an expanded class of point sets. With this extension, except in places where points are highly non-uniformly spaced or extremely sparse, our algorithm will reconstruct an aesthetically pleasing shape.

\section{Results}

\begin{tabular}{|l|r|r|r|r|r|r|r|r|r|}
\hline Point set & $\mathrm{n}$ & $\mathrm{l}$ & $\mathrm{m}$ & $\mathrm{i}$ & Comb. & $s_{g}$ & $s_{l}$ & $\alpha_{\min }$ & $u_{\max }$ \\
\hline Tulip & 79 & 15 & 18 & 2 & $>10^{14}$ & 1 & 12 & $20^{\circ}$ & 4.2 \\
Goose & 74 & 14 & 10 & 1 & $>10^{8}$ & 1 & 4 & $61^{\circ}$ & 3.6 \\
Octopus & 166 & 37 & 20 & 3 & $>10^{35}$ & 4 & 40 & $72^{\circ}$ & 2.7 \\
Crocodile & 129 & 8 & 7 & 1 & 46080 & 2 & 5 & $34^{\circ}$ & 2.5 \\
Elephant & 75 & 15 & 9 & 0 & $>10^{14}$ & 5 & 2 & $13^{\circ}$ & 5.5 \\
Inverted heart & 34 & 5 & 6 & 0 & 3888 & 3 & 1 & $19^{\circ}$ & 3.3 \\
Close curves & 13 & 4 & 10 & 2 & 128 & 3 & 25 & $49^{\circ}$ & 2.1 \\
Random10 & 10 & 4 & 0 & 0 & 270 & 3 & 1 & $40^{\circ}$ & 6.6 \\
Three loops & 18 & 5 & 8 & 0 & 540 & 1 & 1 & $76^{\circ}$ & 2.0 \\
Rail joint & 30 & 3 & 10 & 1 & 9 & 2 & 3 & $122^{\circ}$ & 16.5 \\
Family & 9990 & 33 & 5 & 1 & $>10^{14}$ & 16 & 2 & $45^{\circ}$ & 1.4 \\
\hline
\end{tabular}

Table 4: Complexity table: $n=$ points, $l=$ leaf vertices, $m=$ vertices in largest inflatable sub-tree, $i=$ largest number of interior vertices in such a sub-tree. Combinations gives the number of the terminal nodes (solutions) in the hypothetical complete solution tree. $s_{g}$ is the number of solutions evaluated globally. $s_{l}$ is the maximum number of solutions evaluated locally for any inflatable sub-tree. $\alpha_{\min }$ is the minimum angle. $u_{\max }$ is the largest local non-uniformity factor.

We have implemented this algorithm and tested it with a very large number of sample point data sets. Specifically, we have tested the performance of our algorithm on many of what are considered as problematic point sets, point data for which the 

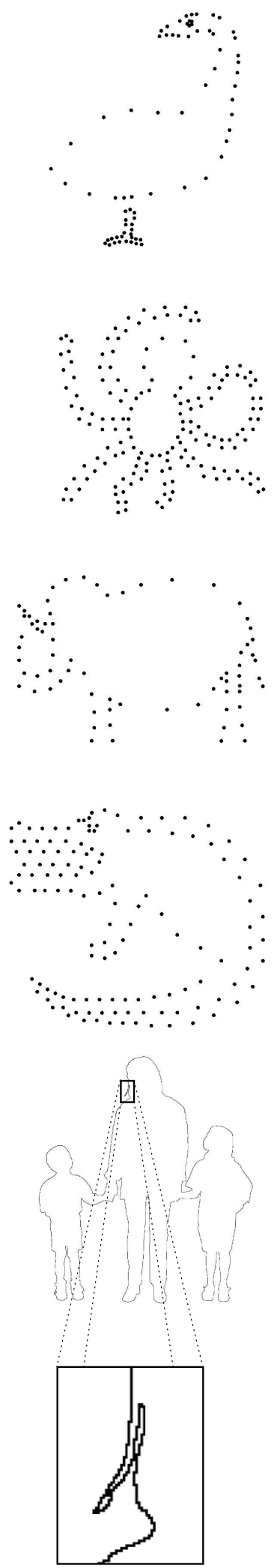
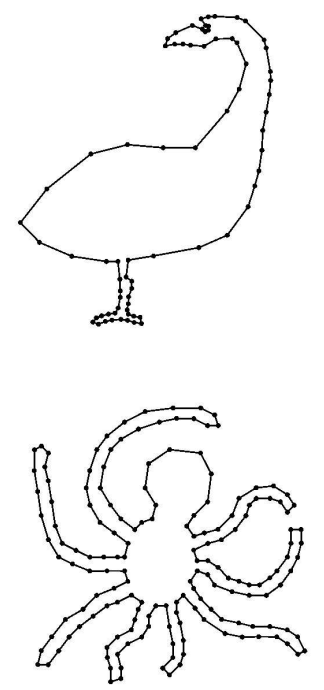

a)
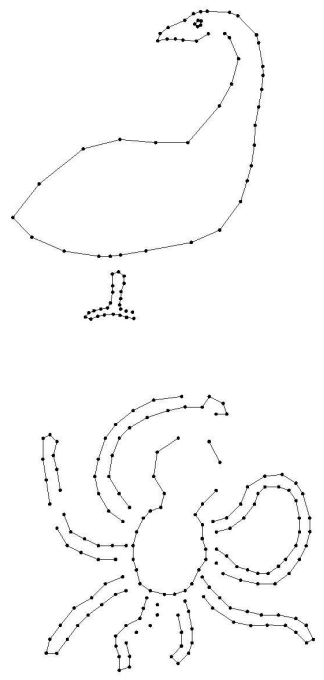

b)
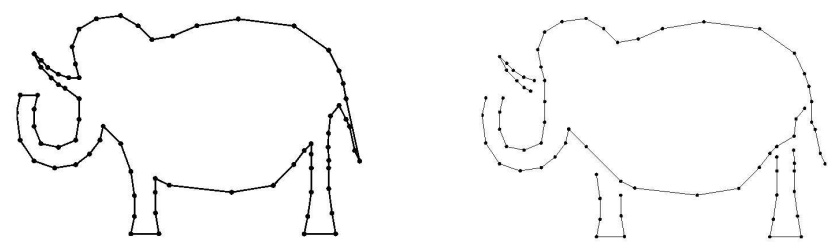

c)
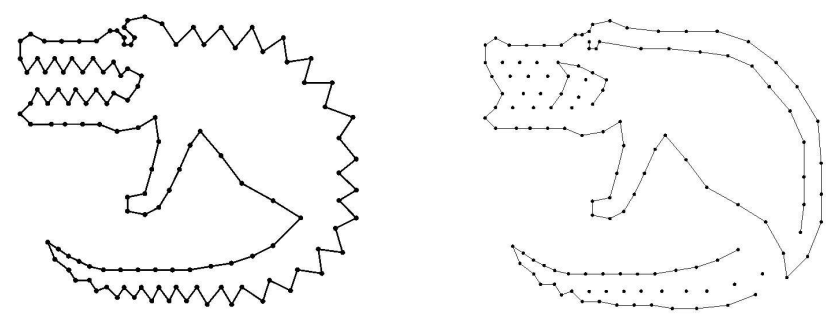

d)

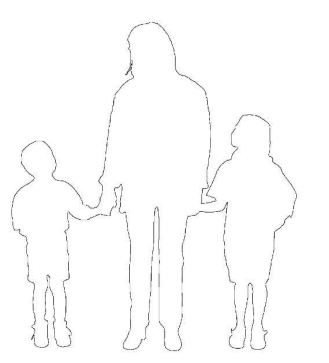

e)
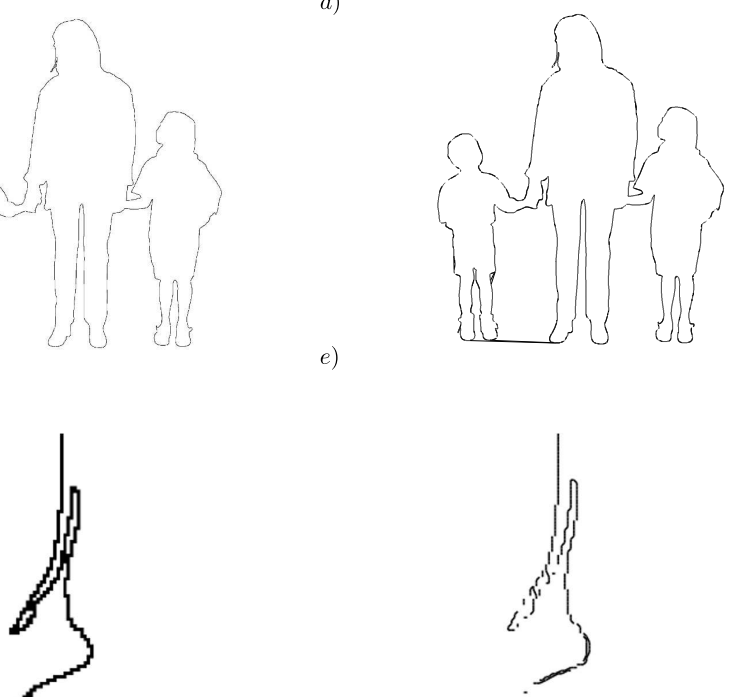
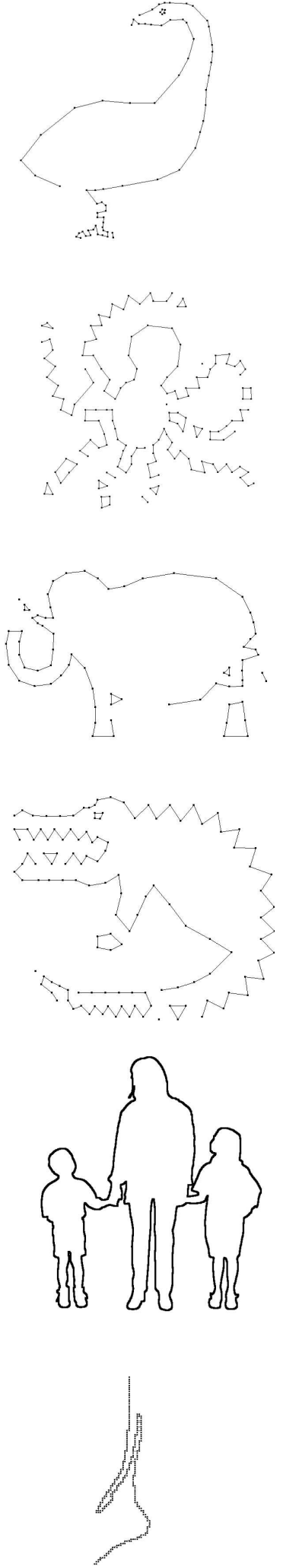

Figure 12: Columns: 1) Point set. 2) Our reconstruction. 3) GathanG with default parameters minAngle = 10 and maxIter = 4. 4) DISCUR. Rows: a) Goose (Amenta et al. [3]). b) Octopus: Close curves with sparse sampling. c) Crocodile: Sharp features. d) Elephant: non-uniform sampling and very sparsely sampled at corners. e) 10k points sampled on silhouette image: only our method produces a manifold. f) Detail of e. 

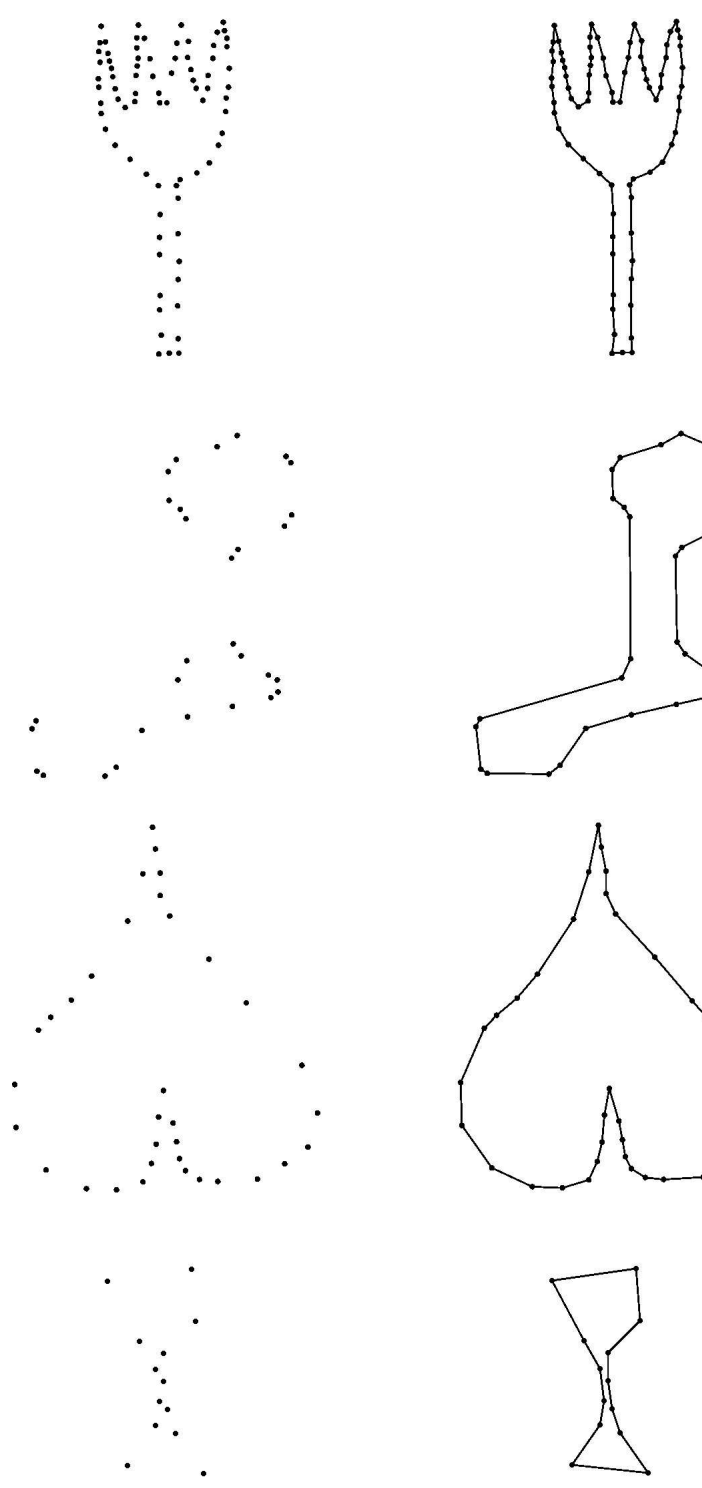

a)
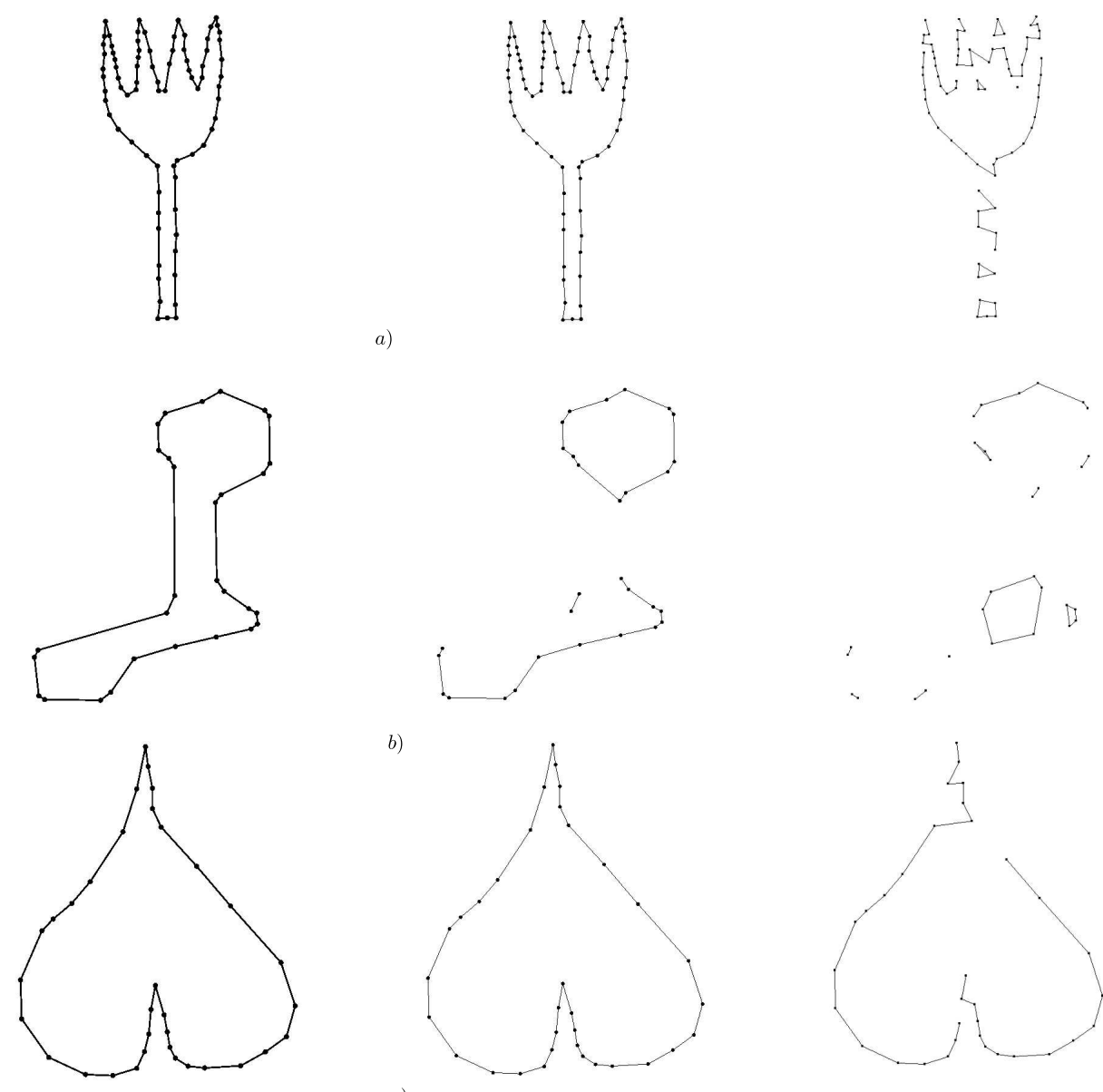

b)
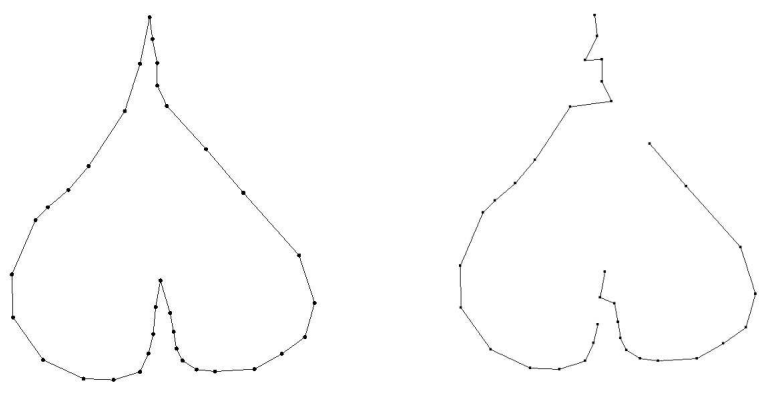

c)

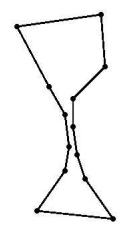

d)
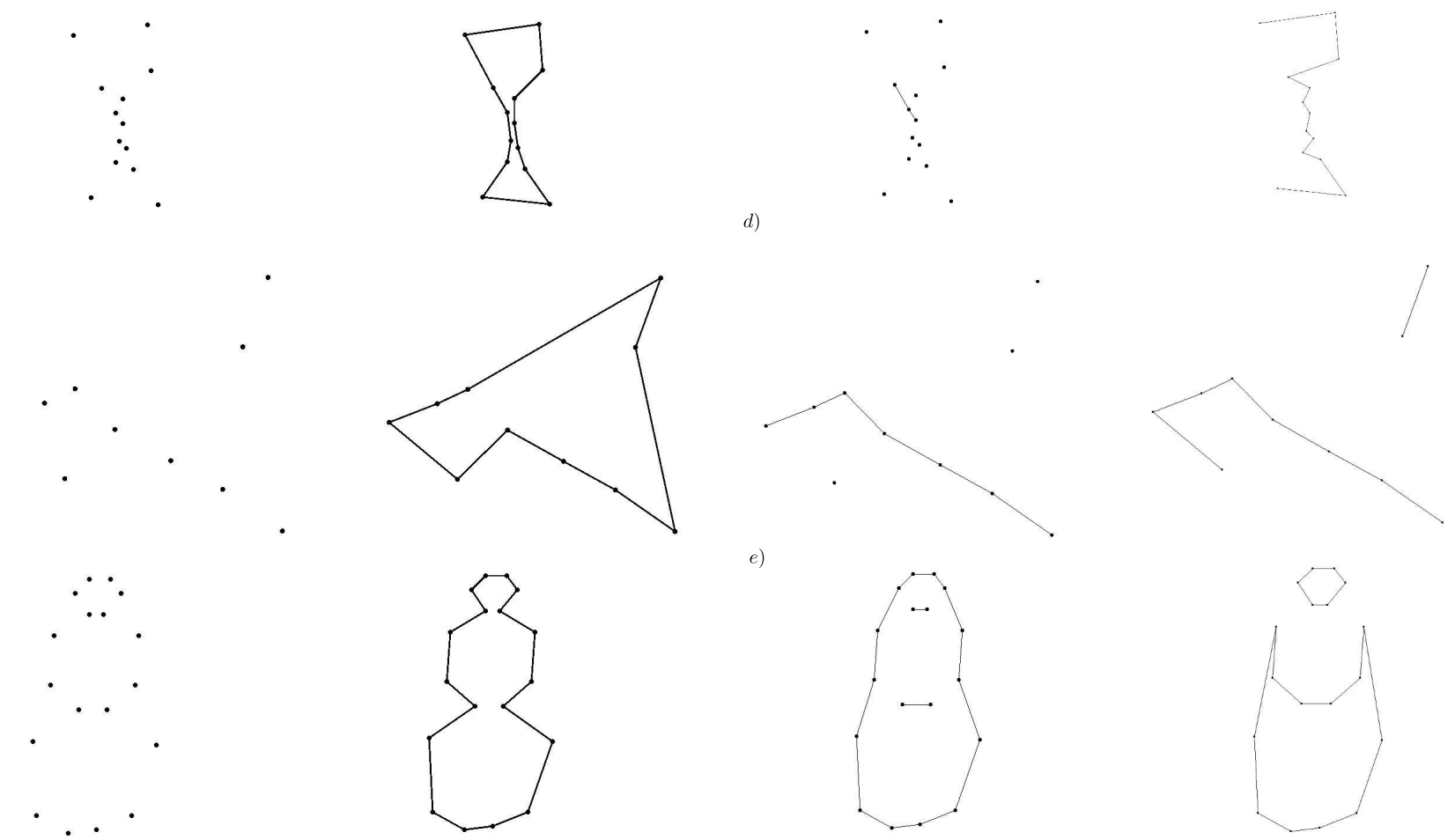

f)

Figure 13: Columns: 1) Point set. 2) Our reconstruction. 3) GathanG with default parameters minAngle $=10$ and maxIter $=4.4)$ DISCUR. Rows: a) Tulip (Althaus and Mehlhorn [1]). b) Rail-joint (engineering part). c) Inverted heart (Zeng et al. [31]). d) Close curves. e) 10 random points. f) Three loops. 
currently best known algorithms fail to produce desirable results. We focus on critical details as typical point sets are likely dense and thus not useful to properly illustrate the advantages of using our method. We compare our results with the previous best reconstruction method, GathanG from [14] (see Figure 12 and Figure 13) and also with DISCUR [31]. The latter have already compared their results with many of the other methods mentioned earlier. We have also compared with other approaches such as $\alpha$-shapes or greedy algorithms (i.e. Boissonnat's sculpturing technique applied to $2 D$ [9]), but we find that these methods settle rather too quickly into local minima and yield poor results for the non-uniform and sparsely sampled cases that we are illustrating in our comparative studies.

In our figures we have excluded the normal well sampled cases for which most methods including ours yield good results. We have mainly included point sets which samplingoriented reconstruction algorithms have not been able to handle correctly and efficiently. This is clear from the examples. Further the results demonstrate that closely spaced shape segments, sharp corners, non-uniform and non-dense sampling are all handled very well by our algorithm. What is a bit surprising to us is the fact that the image silhouette data which is actually dense and uniform in most places could not be handled correctly by the other algorithms. Since we only had access to the executables of other algorithms, we can only show their results using screen shots of the output. Hence problems in connectivity are not always visible for highly dense point sets. We have noted that the total run times for all the algorithms are dominated by Delaunay graph computation time, and hence are all nearly the same. We do not feel that other comparisons such as actual run-times are illustrative. For example, for DISCUR only their binary is available to us. It strictly works with integer coordinates and the implementation is probably not optimized as it becomes very slow even for medium-sized point sets.

It could be argued that our requirement of a single closed shape (guided by the Gestalt law of closure) limits our algorithm's applicability. Where as Crust, Gathan, DISCUR and others are not. And even GathanG, although it is mainly targeted towards closed curves, handles open curves as well. However, the closed or open result from these algorithms has to be user specified or based on requiring the point configuration to satisfy a specified geometric condition, such as limit on point separation distance, abrupt curvature change, etc. In our algorithm, we could always apply the same conditions in a postprocessing operation to remove offending edges and yield an open curve.

Also, if it is known that an open curve is to be generated, Steiner points can be appropriately introduced as user input, although deciding on the location for these Steiner points puts an additional burden on the user. On the other hand, it is important to restate that the imposition of Gestalt's law of closure lets our method realize far better results for sparsely sampled point sets.

Actually, we conjecture that our requirement of closed shape, restriction to edges in $D G$, classification and retention of retained segments and imposition of the manifold condition are what helps us significantly prune the solution space which otherwise has to be explored in full by TSP algorithms.

\subsection{Complexity}

As can be seen in the results section above, actual run time is nowhere near the worst case. However for theoretical completeness, we derive the worst case performance of this algorithm. The worst case is of course for data sets which have completely random distribution of points in 2D space. We first provide definitions of a few parameters needed in the complexity formulation.

- Global solutions $s_{g}$ : denotes the number of calls to apply and eliminate procedure (see start of Section 5).

- Local solutions $s_{l}$ : denotes the maximal number of solutions evaluated in any call to inflate and select minimum loop procedure (see Section 5.1).

Based on a point set with $n$ vertices with $l$ of them being leaf vertices, $S_{\text {min }}$ can be reconstructed in:

$$
O\left(\max \left(s_{g}(n \log n), s_{l}\right)\right)
$$

Below we derive the worst case complexity for the individual steps in the algorithm:

- Create $D G, E M S T$, segment and classify: $\mathrm{O}(\mathrm{n} \log \mathrm{n})$.

- Create segment-candidate association table: In the worst case $\mathrm{O}(\mathrm{nl})$ for the traversal of all $n$ edges of the tree for at most $d l$ candidates $(d=6$ is the average of incident edges for a vertex in $D G$ ). In practice for non-randomly distributed point sets the complexity is lower.

- apply and prune: $\mathrm{O}(\mathrm{n} \log \mathrm{n})$. It can be called $\mathrm{O}\left(c^{r}\right)$ times, where $c$ are the columns and $r$ the rows of the segmentcandidate table.

- inflate and select minimum loop: $\mathrm{O}((\mathrm{m} \log \mathrm{m}) \mathrm{i} !)$, where $i$ is the maximum number of interior vertices in an inflatable segment.

- remove cut edges: $\mathrm{O}(\mathrm{n})$.

Table 4 shows the actual values of these parameters for the various point sets used in Figures 12 and 13.

As can be derived from the global complexity equation above, run-time increase is linearithmic with the number of points, provided that the global factor $s_{g}$ is small w.r.t. $n$ and local $s_{l}$ is small w.r.t. nlogn. This is the case for all figures in Table 4, even for ones which are large, sparse and non-uniform at the same time. These factors become large only when the point set configuration is extreme in sparseness or non-uniformity of point spacing.

\section{Conclusion and Future Work}

We have presented a powerful and efficient algorithm which is capable of reconstructing aesthetically pleasing single closed interpolating 2D shape for an unorganised point set without requiring highly dense or uniform sampling. The results are better 

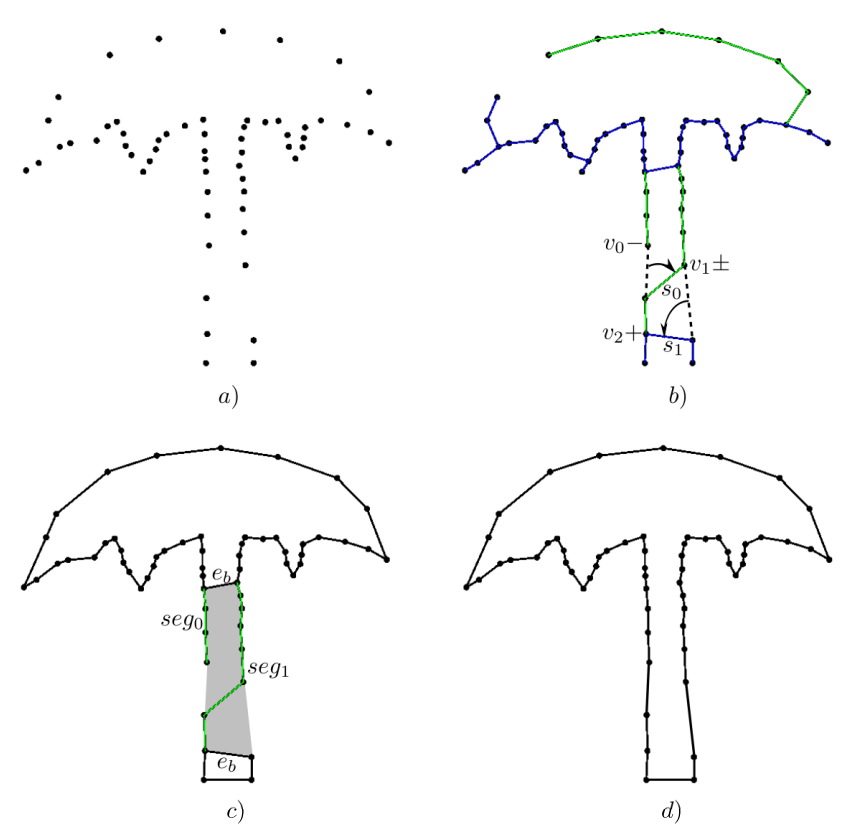

Figure 14: a) The non-conforming mushroom point set (Dey and Wenger [13]). b) EMST with segments (blue: non-retained, green: retained) marked. The two snaps $s_{0}, s_{1}$ share an impact at $v_{1}$ but their $e_{+}$are not contained in one segment, contrary to the required condition. c) The consequence is incomplete reconstruction at those snaps' affected segments: the two retained segments $s e g_{0}, \operatorname{seg}_{1}$ remain without candidates. We can extend our algorithm to consider the envelope of their combined point set, including the base edges $e_{b}$, shaded grey, and then carry out the inflate operation to yield the desired shape. d) $S_{\min }$.

than those of all other known solutions for this $2 \mathrm{D}$ reconstruction problem. The actual run-time complexity statistics demonstrate that it is linearithmic for most practical cases.

Our algorithm does not employ any user-specified parameters and it does not require a sampling criterion. It does have a limitation for the point configuration which is mainly a safeguard to avoid very badly spaced points.

We also note that the number of leaf vertices in the EMST of a point set correlates well with the running time required for the reconstruction of its $S_{\min }$.

For another class of point sets which fall in the nonconforming category as they fail our required condition, i.e. as in the case seen in Figure 14 we show a potential extension.

Further, we believe that the primary methodology of starting with a skeleton shape and then transforming it into the final interpolating shape can be extended to $3 \mathrm{D}$. We are presently making progress on the formulation of such an extension for 3D shape reconstruction, a much more difficult problem.

\section{Acknowledgements}

We are very grateful to the anonymous referees of the earlier versions for their insightful comments, which have helped immensely to improve the presentation in this version. This research was supported through an NSERC Discovery Grant, NCE Grand research grant, Engineering and Computer Science Faculty Research Grants and Canada Foundation for Innovation which supported equipment acquisition. We are grateful to
Prof. T. K. Dey and Prof. Y. Zeng for providing us the code for their algorithms which we have used in our comparison study.

\section{References}

[1] Althaus, E., Mehlhorn, K., 2000. Tsp-based curve reconstruction in polynomial time. In: Proc. 11th ACM-SIAM SODA'00. pp. 686-695.

[2] Althaus, E., Mehlhorn, K., Schirra, S., 2000. Experiments on curve reconstruction. In: Proc. 2nd Workshop Algorithm Eng. Exper. pp. 103-114.

[3] Amenta, N., Bern, M., Eppstein, D., 1998. The crust and the $\beta$-skeleton: Combinatorial curve reconstruction. Graph. Models and Im. Proc 60 (2), 125-135.

[4] Applegate, D., Bixby, R., Chvtal, V., Cook, W., 2011 (accessed Mar 8, 2011). Concorde TSP Solver.

URL http://www.tsp.gatech.edu/concorde.html

[5] Arora, S., 1996. Polynomial time approximation schemes for euclidean traveling salesman and other geometric problems. In: Journal of the ACM. pp. 2-11.

[6] Attali, D., 1997. $\mathrm{r}$-regular shape reconstruction from unorganized points. In: Symposium on Computational Geometry. pp. 248-253.

[7] Attene, M., Spagnuolo, M., 2000. Automatic surface reconstruction from point sets in space. Computer Graphics Forum 19 (3), 457-465.

[8] Bernardini, F., Bajaj, C. L., 1997. Sampling and reconstructing manifolds using alpha-shapes. In: In Proc. 9th Canad. Conf. Comput. Geom. p. 0.

[9] Boissonnat, J.-D., 1984. Geometric structures for three-dimensional shape representation. ACM Trans. Graph. 3 (4), 266-286.

[10] Buchin, K., Knauer, C., Kriegel, K., Schulz, A., Seidel, R., 2007. On the Number of Cycles in Planar Graphs. Computing and Combinatorics, 97-107.

[11] Dey, T. K., Kumar, P., 1999. A simple provable algorithm for curve reconstruction. In: Proc. 10th ACM-SIAM SODA '99. pp. 893-894.

[12] Dey, T. K., Mehlhorn, K., Ramos, E. A., 1999. Curve reconstruction: Connecting dots with good reason. In Proc. 15th ACM Symp. Comp. Geom 15, 229-244.

[13] Dey, T. K., Wenger, R., 2001. Reconstructing curves with sharp corners. Computational Geometry 19 (2-3), 89 - 99.

[14] Dey, T. K., Wenger, R., 2002. Fast reconstruction of curves with sharp corners. Int. J. Comput. Geometry Appl. 12 (5), 353 - 400.

[15] Dillencourt, M. B., 1987. A non-hamiltonian, nondegenerate delaunay triangulation. Inf. Process. Lett. 25 (3), 149-151.

[16] Edelsbrunner, H., Kirkpatrick, D. G., Seidel, R., 1983. On the shape of a set of points in the plane. IEEE Trans. Inf. Theor. IT-29 (4), 551-559.

[17] Figueiredo, L. H. d., Mirandas Gomes, J. d., 1994. Computational morphology of curves. The Visual computer 11 (2), 105-112.

[18] Genoud, T., 1990. Etude du caractère hamiltonien de delaunays aléatoires, travail de semestre.

[19] Giesen, J., 1999. Curve reconstruction in arbitrary dimension and the traveling salesman problem. In: Proc. 8th DCGI '99. pp. 164-176.

[20] Glanvill, M., Broughan, K., 1997. Curve and surface reconstruction in r2 and r3. In: HPC-ASIA '97: Proceedings of the High-Performance Computing on the Information Superhighway, HPC-Asia '97. IEEE Computer Society, Washington, DC, USA, p. 395.

[21] Grigni, M., Koutsoupias, E., Papadimitriou, C., 1995. An approximation scheme for planar graph tsp. In: Proceedings of the 36th Annual Symposium on Foundations of Computer Science. FOCS '95. IEEE Computer Society, Washington, DC, USA, pp. 640-.

[22] Helsgaun, K., 2009. General k-opt submoves for the linkernighan tsp heuristic. Mathematical Programming Computation 1 (2-3), 119 - 163.

[23] Hoos, H. H., 2009. On the empirical scaling of run-time for finding optimal solutions to the traveling salesman problem. Technical Report 17, University of British Columbia, Department of Computer Science.

[24] Kirkpatrick, D. G., Radke, J. D., 1985. A framework for computational morphology. Computational Geometry, 217-248.

[25] Kruskal, Joseph B., J., 1956. On the shortest spanning subtree of a graph and the traveling salesman problem. Proc. of the American Mathematical Society 7 (1), 48-50.

[26] Lee, I.-K., 1999. Curve reconstruction from unorganized points. Computer Aided Geometric Design 17 (2), 161 - 177. URL http://www.sciencedirect.com/science/article/pii/S016783969

[27] Mather, G., 2008. Foundations of perception. In: CAD. pp. 210-222. 
[28] Nguyen, T. A., Zeng, Y., 2008. Vicur: A human-vision-based algorithm for curve reconstruction. Robotics and Computer-Integrated Manufacturing 24 (6), 824 - 834, fAIM 2007, 17th International Conference on Flexible Automation and Intelligent Manufacturing.

[29] Savelsbergh, M., Volgenant, T., 1985. Edge exchanges in the degreeconstrained minimum spanning tree problem. Computers \& Operations Research 12 (4), 341 - 348.

[30] Veltkamp, R. C., 1993. 3D computational morphology. Computer Graphics Forum (Eurographics '93) 12 (3), 115-127.

[31] Zeng, Y., Nguyen, T. A., Yan, B., Li, S., 2008. A distance-based parameter free algorithm for curve reconstruction. CAD 40 (2), 210-222. 